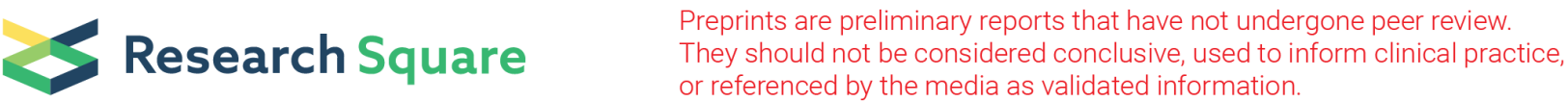

\section{The first awake simultaneous PET-MR study of an adult with fragile $X$ syndrome: $A$ case report}

Soujanya Gade ( $\nabla$ soujanyagade@gmail.com )

Stanford University https://orcid.org/0000-0002-0057-8289

Trine Hjørnevik

Oslo University Hospital: Oslo Universitetssykehus

Jun Hyung Park

Stanford University School of Medicine

Bin Shen

Stanford University School of Medicine

Meng Gu

Stanford University School of Medicine

Jessica Tseng

Stanford University School of Medicine

Daniel Barbosa

Stanford University School of Medicine

Christoph Leuze

Stanford University School of Medicine

Sharon Pitteri

Stanford University School of Medicine

Jaeho Jung

BIK Therapeutics

Byung Chul Lee

Seoul National University

Sang Eun Kim

Seoul National University Hospital

Jennifer McNab

Stanford University School of Medicine

Daniel Spielman

Stanford University School of Medicine

Scott S. Hall

Stanford University School of Medicine

Lawrence K. Fung

Stanford University School of Medicine

Frederick T. Chin 


\section{Case report}

Keywords: Simultaneous PET-MR, fragile x syndrome, GABAA receptors, neurodevelopmental disorders, awake

Posted Date: February 3rd, 2021

DOI: https://doi.org/10.21203/rs.3.rs-189580/v1

License: (1) This work is licensed under a Creative Commons Attribution 4.0 International License. Read Full License 


\section{Abstract}

Introduction: Fragile X syndrome (FXS) is a debilitating neurogenetic disorder that can result in a multitude of impairments in cognition, memory, and learning.

Case Presentation: a 25-year-old male with FXS participated in this study. The participant obtained scores in the non-spectrum range on the Autism Diagnostic Observation Scale and obtained an full scale IQ score of 57 (Verbal IQ = 23 and Nonverbal IQ =34) on the Stanford-Binet Intelligence Scales $(S B-5)$. On the Vineland Adaptive Behavior Scales, 2nd Edition (VABS-2) he obtained a composite score of 66. Pre-scan serum cortisol reactivity was $16.45 \mathrm{mcg} / \mathrm{dL}$. Following a $\left[{ }^{18} \mathrm{~F}\right]$ flumazenil $(5 \mathrm{mCi})$ intravenous bolus injection, the participant was scanned without sedation on a hybrid PET-MR system (Signa, GE Healthcare, Waukesha, WI) for 60 mins. List mode PET data, structural and diffusion MRI (DWI), and MR spectroscopy (MRS) were acquired simultaneously. Quantitative PET and DWI measures were extracted from 83 pre-defined regions of interest. MRS data was collected from two $20 \mathrm{cc}$ voxels (thalamus and dorsolateral prefrontal cortex).

Conclusion: This is the first study to investigate neuromolecular behavior in FXS without the use of sedation using PET-MR. Mapping the neuromolecular differences in FXS can lead to targeted treatments that can significantly improve quality of life for families and individuals with intellectual disabilities.

\section{Background}

Fragile $\mathrm{X}$ syndrome (FXS) is an X-linked genetic condition that is the result of a mutation at the Xq27.3 fragile site resulting in the loss of function of the FMR1 gene caused by an expansion of the CGG trinucleotide in the 5'untranslated region (Verkerk et al., 1991). Approximately 1 in every 430 males carry the fragile $X$ mutation and it is the leading heritable cause of intellectual disability (ID) (Tassone et al., 2012; Hunter et al., 2014). Males with FXS have a phenotype characterized by a long, narrow face, large protruding, ears, prominent jaw and forehead, flat feet, unusually flexible fingers and macroorchidism (The National Institue of Mental Health, 2020).

The behavioral phenotype of FXS includes autistic-like behaviors and deficits in executive function, memory, and visuospatial reasoning (Backes et al., 2000). The developmental consequence to these impairments becomes evident in childhood and continue through adulthood (Bailey et al., 2001). Often a diagnosis of FXS coincides with a diagnosis of autism (Thurman et al., 2015). Similar to case profiles of autism a range of variation from individual to individual can be observed across both genders.

Currently, up to $70 \%$ of clinical interventions for FXS implement the use of prescription medications where pharmacological agents such as antipsychotics, antidepressants, benzodiazepines and mood stabilizers are recommended in conjunction with behavioral intervention and focused on symptomatic treatment (Berry-Kravis \& Potanos, 2004; Laxman et al., 2018). These agents have significant side effects such as nausea, tachycardia, diaphoresis, akathisia, weight gain and lethargy (Laxman et al., 2018). Although these treatments are available for supportive care, there is no single treatment for FXS in which 
pharmacological agents are administered for targeted treatment of the affected systems in FXS (BerryKravis et al., 2011; Berry-Kravis \& Potanos, 2004). A major barrier for targeted treatment is attributed to the underlying uncertainty due to the lack of understanding of brain structure, physiology and functions.

Recently, research has implicated gamma-aminobutyric acid (GABA) system in the role of learning and memory (Mihalek et al., 1999). Specifically, GABAergic dysfunction has been examined in autism and shown to play a key role in normal neuronal function (Chao et al., 2010; Ma et al., 2005). Evidence indicates $G_{A B A}$ receptors are involved in the inhibitory mechanisms in the brain that give rise to the behavioral symptoms that can be observed in autism (Coghlan et al., 2012). Although there have been animal studies investigating $\mathrm{GABA}_{A}$ in $\mathrm{FXS}$, there has not yet been any development of information regarding the $\mathrm{GABA}_{A}$ receptor availability in human adults with $\mathrm{FXS}$. Understating the differences in neuro-mechanisms in relation to the behavioral symptomology can serve to be useful in understanding the etiology of the disorder as well as tailoring targeted treatments for patients with FXS.

Molecular imaging methods such as positron emission tomography (PET) and magnetic resonance (MR) imaging have substantially contributed in the last decades to deepen our knowledge on brain functioning in many neurological conditions and more recently in FXS (James Frost et al., 1986). PET is the most effective method to estimate receptor occupancy rates of pharmacological agents in the brain (D'Hulst et al., 2015; James Frost et al., 1986). Multimodal imaging strategies, such as simultaneous PET with MRI, are providing researchers a new way to visualize disease non-invasively with less time used for scanning (James Frost et al., 1986). Although the use of MRI and fMRI has been utilized to investigate the structure of the FXS brain, PET imaging has only been employed within this population with the use of sedation (Bruno et al., 2017; Hall, Dougherty, \& Reiss, 2016; Hoeft et al., 2010, D’Hulst et al., 2015). However, anesthetic agents have been shown to influence the uptake and binding of GABA receptors leading to potentially inaccurate measurements (Palner et al., 2016). The use of PET-MR can help identify dysfunction on a neurotransmitter level and therefore provide insight for drug treatment that is targeted to accommodate these deficits rather than aim for symptomatic treatment based on behavior. The challenge in utilizing this technology arises with patient populations such as FXS that often are unable to tolerate scanning.

The use of MRI and fMRI has been utilized to investigate the structure of the FXS brain often with the use of anesthesia. Studies that aim to implement brain imaging protocols are only able to successfully scan participants with FXS with the use of anesthesia. This means, any study utilizing MRI has to admit their subjects to a hospital where they are given general anesthesia for the duration of the MRI. Furthermore, the resulting data yields information about volume and structure of the brain but not about neurotransmitter behavior (Bruno et al., 2017; Hall, Dougherty, \& Reiss, 2016; Hoeft et al., 2010, D'Hulst et al., 2015). Thus far, no study has employed the use of PET technology without sedation in studying GABAergic behavior in FXS. Recent studies have had to employ the use of mild sedatives for PET data acquisition even after MRI training and were only successful in scanning some participants (Brašić et al., 2020). This is primarily due to the challenges involved in acquiring a scan in which the patient lies still in a tight space while being exposed to loud sounds from the magnet for a whole hour. Furthermore, the 
lack of language skills, high levels of anxiety in patients with FXS and the requirement of an intravenous tracer to complete a PET scan add to the demand of the task. Due to these fundamental challenges, studies involving a brain imaging protocols commonly use the approach of administering anesthesia, however, this study implemented, a never-been-used, innovative approach of cognitive-behavioral training techniques in place of sedation. This study is the first of its kind to successfully achieve PET-MR scanning with intravenous injections of $\left[{ }^{18} \mathrm{~F}\right]$ flumazenil without the use of sedation in FXS.

Here, we present a case study of a 25-year-old male with full mutation FXS that underwent a gradedexposure MRI behavioral training program and was able to successfully complete a 60-minute PET-MR examination.

\section{Methods}

\section{Screening and Enrollment.}

This study is part of a larger study NIH funded research study at Stanford University. Exclusionary criteria included any contraindication for MRI scanning procedures (metal in body, braces, claustrophobia, etc.). Eligibility was assessed with a detailed intake questionnaire and included screening for substance abuse, psychiatric conditions (bipolar disorder or schizophrenia), traumatic brain injury and BMI less than 18.5 for eligibility (see supplementary table). The estimated completion time for this interview was approximately 30 minutes. Once the participant met the eligibility criteria, an electronic consent from was completed before proceeding to the MRI preparation and training (see Table 1 for activities list).

\section{PET-MR Scan Preparation.}

Due to the difficulty in acquiring successful scans of participants with FXS, a detailed preparation plan was prepared. Prior to their visit, the participant underwent a thorough screeing with a questionnaire for the ability to comply with the image acquisition procedures. The first step in training involved a desensitization process in which the participant reviewed two different videos of an adult preparing and undergoing a PET-MR scan. The media was open-source material found online and included footage of the PET-MR equipment and associated noises, descriptions of what they will be asked to do. The participant then met with the study staff member via videocall to discuss and address any anxieties from the materials he reviewed. This session was also utilized to build rapport between the participant and the study staff member who would be conducting all the psychosocial assessments in addition to the scan during the planned visit.

The second video included scenes of a person receiving antecubital injection prior to entering the scanner and examples of experimental stimuli that may be viewed, and the images being acquired. After viewing this second video, the study staff member conducted a follow-up video session to reviewed and debrief about the steps involved. 
Following the two video viewings, the participant was asked to listen to a 20-minute streaming audio that played a collection of MRI sounds that are associated with different pulse sequences that they would is likely to hear during their scans. Prior to listening to the audio clips, the study staff member narrated information pertaining to the PET-MR experience and set expectations for the participant's performance. This step was designed to serve as exposure to the new stimuli and give the participant an opportunity to address anxieties. Additionally, it provided the staff member with insight on potential triggers to avoid as well as coping mechanisms that could help reduce anxiety during the day of the scan.

The next behavior training session was structured as a "game" with a parent present to help as needed. The staff member worked with the parent and participant via a video call to create an at-home-MRI simulation environment. The participant was told that the game rules were to build a fort and see how long they could hold still while laying inside the fort. The participant's parent constructed a small fort-like space on the floor of the living room using a chair and blankets. This "fort" was used to practice laying still for 3 incrementally increasing periods of time (5, 10, 15 minutes). The participant completed "playing the game" by lying motionless on the floor while the staff member guided and timed them via the videocall. Once he were able to complete 10 minutes without moving, he would then jump to the next level of the game. This level involved his parent placing pillows firmly on either side of his head and playing the audio track of MRI sounds through headphones. This level of the game was played until a successful 15 minute session of holding still was completed.

One week before their planned visit, the study staff member met with the participant via videocall once again and reviewed all the steps for the scan. This meeting was also utilized to continue to build rapport, answer any questions and give the participants a sense of autonomy when undertaking a new and unfamiliar task such of the MRI.

Lastly, a final check in was done via phone call before the participant and his mother flew to the study site. Once the participant arrived at the study site with their parent for their visit, they participated in a final practice MRI session one day before their scheduled scan. The simulator on site was designed to train research participants to cooperate with the motion control requirements of PET-MR. During this session, additional behavioral techniques were implemented to counter anxiety experienced in association with the equipment and procedures. This step involved duplication and control of the salient stimuli in the imaging environment, gradual exposure to the equipment, awareness around sensations involved during image acquisition, and reinforcement of the participant's positive coping skills and efforts to inhibit body motion when instructed. These procedures are implemented with computer-assisted measurement and feedback for head motion over a 1-hour period for each participant.

Additionally, the PET-MR scanner was equipped with video watching capabilities and so the participants were able to pick and watch their favorite movie during the scan process. This was a critical factor in increasing the ability of the participants to tolerate the long scan times as it reduced anxiety and provided distraction. 


\section{Case Presentation}

\section{Study Participant.}

A 25-year-old Caucasian, non-Hispanic male accompanied by his mother were flown in for a two-day visit at the study site. The participant and his caregiver landed late afternoon in the city of the study site. Upon arrival, they drove to the study site and completed all consent forms for the study. The following two days were dedicated to the study protocol that included behavioral training, neuropsychological assessments and the PET-MR scan.

There was no history of maternal fever, drug or other teratogens during pregnancy. Genetic testing for FXS was conducted at age three after a speech and occupational therapy evaluation. FMR1 DNA test results indicated that he is a full mutation (> 300 repeats). The participant did not have any contraindication for MRI scanning procedures (metal in body, braces, claustrophobia, etc.). No history of substance abuse, psychiatric conditions (bipolar disorder or schizophrenia), traumatic brain injury and BMI less than 18.5 was reported.

\section{Psychometric Measures.}

The behavioral assessments included a battery of standardized assessments of adaptive behavior, autistic symptomatology and cognitive ability. Standardized assessments of the individual were conducted to measure adaptive skill levels using the Vineland Adaptive Behavior Scales, 2nd Edition (VABS-II) and degree of autistic symptomatology using the Autism Diagnostic Observation Schedule, 2nd Edition (ADOS-2).

Adaptive behaviors encompass a set of observable and functional skills that allow individuals to respond and meet environmental and social demands. The lack of development in any of the subdomains of adaptive functioning typically necessitates some level of dependence on a caregiver/parent. The adaptive profiles can give better insight into functional ability over IQ scores particularly for individuals neurodevelopmental disorders such as fragile X syndrome (FXS). The Vineland Behavioral Rating Scales Analysis 2nd Edition (VABS-2) was used to measure behavior in three major domains, communication, daily living skills, and socialization. The participant scored 59 in the adaptive behavior composite, which indicates moderately deficient adaptive behavior. The composite score is below the normative mean of 100. The Stanford-Binet Intelligence Scales (SB-5) is a standardized intelligence test that can be used for ages 2-85 + years and provides a full-scale IQ composite score (general composite), verbal (VIQ) and non-verbal IQ (NVIQ) and five core cluster scores: Fluid, Knowledge, Quantitative, Visual-Spatial, and Working Memory. The obtained a standard score of 42 ( $<.1$ percentile rank) on the composite which falls in the moderately impaired range. This score is consistent with the typical presentation of FXS. He scored slightly higher in the non-verbal IQ portion of the assessment. He did not meet classification on the Autism Diagnostic Observation Scale (ADOS-2), adult module.

\section{Blood Sampling.}


Blood samples from the participant were collected in 4.5mL, BD Vacutainer, glass "red-top" tubes prior to intravenous injection of clinical grade $\left[{ }^{18} \mathrm{~F}\right]$ flumazenil. The sample was allowed to clot at room temperature for up to one hour and centrifuged at $4^{\circ} \mathrm{C}$ for $10 \mathrm{~min}$ at $1100 \mathrm{~g}$. Upper phase sera were aliquoted ( $200 \mu \mathrm{l}$ per assay tube) and stored at $-80^{\circ} \mathrm{C}$ until processing. The cortisol level $(16.45 \mathrm{mcg} / \mathrm{dL})$ was measured using a cortisol parameter assay kit (R\&D Systems) pre-scan; this score falls within the normal reference range of cortisol adults.

\section{Imaging.}

PET

A 60-minute scan on time-of-flight (TOF) PET-MR imaging system (SIGNA PET-MR GE Healthcare) was conducted after intravenous injection of $5 \mathrm{mCi}\left[{ }^{18} \mathrm{~F}\right]$ flumazenil. The precursor for $\left[{ }^{18} \mathrm{~F}\right]$ flumazenil synthesis was provided by BIK Therapeutics Inc. (Seongnam, South Korea). No anesthesia or sedatives were used to conduct this scan. The participant received individualized behavior training over the course of four weeks to ensure successful scanning.

The PET listmode data was dynamically reconstructed into 27-time frames using a fully 3D time-of-flight iterative ordered subsets expectation maximization algorithm ( $30 \mathrm{~cm}$ FOV, 28 subsets, 2 iterations, $4 \mathrm{~mm}$ Gaussian filter, 256x256 matrix), and corrected for attenuation, scatter, point spread function, dead time, and decay. MR-based attenuation correction was performed with the clinical atlas-based method as implemented by the manufacturer, where the 2-point Dixon MR images are registered to a CT-based head atlas.

Any patient movement during scanning was corrected for by frame-to-frame alignment using the first 2minute summed data as reference. PET image data were spatially normalized to Montreal Neurological Institute (MNI) space according to the maximum probability atlas method implemented in PMOD 3.7 (PMOD Technologies LCC). The individual T1-weighted MR images were segmented into grey matter probability maps and spatially normalized to the N30R83 maximum probability atlas (Hammers et al., 2003) which contains 83 brain structures. Time-activity curves (TACs) were extracted using pre-defined volumes-of-interest (VOIs) for thalamus, caudate and pons and a reference tissue model (Ichise model; MRTM0; Ichise \& Ballinger., 1996) was used to calculate binding potentials $\left(\mathrm{BP}_{\mathrm{ND}}\right)$ with pons as the reference region. Figure 1 shows a parametric image of specific binding of $G_{A B A}$ receptor $\left[{ }^{18} \mathrm{~F}\right]$ flumazenil to $\mathrm{GABA}_{A}$ receptors. TACs for four selected regions of interest: middle frontal gyrus, caudate nucleus, thalamus and pons are shown in Fig. 2. $\mathrm{BP}_{\mathrm{ND}}$ values for all regions of interest are shown in Table 2.

\section{MRI}

MRI acquisition included T1- and T2-weighted images, MEGA-SPECIAL GABA-editing sequence and Diffusion-weighted Imaging (DWI).

\section{MRS}


MRS data were acquired using a 32-channel head coil on a GE PET-MR 3T scanner using an improved MEGA-SPECIAL sequence for GABA editing with robust suppression of co-edited macromolecules. MRS data were acquired simultaneously with the PET data acquisition. Data from two $20 \mathrm{cc}$ voxels, one in the thalamus and the other in the DLPFC, were acquired with TE/TR $=80 \mathrm{~ms} / 2 \mathrm{~s}, 15 \mathrm{~min}$ acquisition per voxel. Figure 3 shows the voxel locations, spectra with GABA editing ON and OFF and the edited spectra.

DWI

T1-weighted images were acquired using a three-dimensional (3D) gradient recalled (GR) sequence. Diffusion-weighted images were acquired using a two-dimensional (2D) spin-echo echo-planar imaging (SE-EPI) sequence with the following parameters: repetition time (TR)/echo time (TE): 12,691/94.6 ms, flip angle: $90^{\circ}$, FOV: $240 \times 240 \mathrm{~mm}$, slice thickness: $2.5 \mathrm{~mm}, 60$ directions $\left(b=1000 \mathrm{~s} / \mathrm{mm}^{2}\right)$ and six T2weighted images $\left(b=0 \mathrm{~s} / \mathrm{mm}^{2}\right)$.

Pre-processing was conducted using tools within the FMRIB software library (FSL, Oxford, UK; Smith et al., 2004, 2006). FSL's topup tool was used to estimate susceptibility induced off-resonance fields and correct them. FSL's brain extraction tool (bet) was used to segment and strip the skull from the images. FSL's eddy was used to correct for motion and eddy current artifacts produced from the rapidly changing magnetic fields in MRI. FSL's dtifit was used to fit a diffusion tensor model at each voxel and create fractional anisotropy (FA) and mean diffusivity (MD) maps. FSL's fslstats was used to calculate mean FA and MD based on voxel values within 83 regions of interest, as defined by the N30R83 maximum probability atlas; Gousias et al., 2008), and white matter. Registration of regions of interest from MNI152 $1 \mathrm{~mm}$ asymmetrical space to the subject's native space was performed using Advanced Normalization Tools (ANTs) (Avants et al, 2009), which consists of two successive steps of linear and nonlinear registration between the subject's brain and the MNI brain. In a third step, the MNI-defined ROIs were registered to the subject's space.

We were able to overlay the same regions of interest in which we measured the BPND of $\left[{ }^{18} \mathrm{~F}\right]$ flumazenil to the subject's maps of FA and MD (Fig. 4). The mean FA and MD values observed in these regions of interest are shown in Table 2.

\section{Discussion And Conclusions}

In comparing the participant's VABS-2 score as reported by his parent, the male showed higher levels of verbal communication for the duration of the study. This indicates that his adaptive abilities were likely under-reported by his caregiver. Though he falls in the mildly impaired/delayed range for IQ testing $(57,<$ 0.1 percentile), he did not struggle with attending and following directions, inhibiting taking or moving during the scan. He was able to articulate simple questions about, thoughts and opinion such as discussion about his favorite movies and other hobbies. He was able to make his needs known with simple 3-4-word sentences. He expressed feeling nervous and needed soothing and support from both the staff member and his parent during the scanning process. 
Examining the neuromolecular differences in the clinical populations that are affected with chronic conditions like FXS can lead to better treatment alternatives as well as a breakthrough for a cure. However, achieving a close up look at the affected brain in this population can be quite challenging because adults with FXS often struggle with symptoms such as anxiety and difficulty with self-control. For this reason, this study highlights the importance of the challenges and details behavioral strategies and protocols that can be utilized to overcome the barriers to studying this population. Future studies can utilize these techniques and develop similar trainings to improve feasibility of successfully scanning patients with FXS or other disorder that present with behavioral challenges. Overcoming the barrier of awake scanning for an extended period of time can allow for further analyses that adopt the approach of the involvement of the neuromolecular systems may help elucidate the observable behavioral and cognitive impairments in FXS but will need to have robust planning and resources to support the clinical needs of this population.

This study requires participants to complete a PET-MR scan that takes approximately 60 minutes. Though participants in this study have cognitive impairments, there could be some selection bias due only enrolling participants that can complete the scans. Furthermore, due to prevalence rates, participants flew into participant in the study. The culture as well as available treatments locally as compared to nationally may be a confounding variable that could influence differences between groups

The conceptual understanding of cognitive impact and the GABAergic system can help determine the types of services that are needed to design more effective and targeted treatment plans. This study can serve to further support a relationship in the cognitive profiles and neurological mechanics, particularly within this disorder. Understanding the neural underpinnings of cognitive development is crucial in tailoring individualized interventions to improve developmental outcomes for individuals with FXS as well as other neurodevelopmental disorders. This comprehensive study including imaging and behavioral evaluations is a first of its kind to scan without the use of sedation to establish $G_{A B A}$ behavior in the population of FXS. To date, we have yet to identify brain networks in FXS therefore systematic mapping of the fragile $X$ brain on a molecular level can prove to be groundbreaking toward identifying affected brain circuits. The current study encompasses a global report of one individual across multiple domains ranging from cognitive, behavioral, clinical lab and neuroimaging testing to report preliminary analyses of the study. Future efforts include an expanded study of a larger cohort of FXS participants and a comparison group of IQ matched idiopathic developmental delay is underway.

\section{List Of Abbreviations}

FXS: fragile $X$ syndrome

ID: intellectual disability

GABA: gamma-aminobutyric acid

DLPFC: dorsolateral prefrontal cortex 
ADOS: Autism Diagnostic Observation Scale

IQ: Intelligence Quotient

SB-5: Stanford-Binet Intelligence Scales

VABS-2: Vineland Adaptive Behavior Scales, $2^{\text {nd }}$ Edition

PET: positron emission tomography

MRS: magnetic resonance spectroscopy

DWl: diffusion weighted imaging

BMI: body mass index

FA: fractional anisotropy

\section{Declarations}

Acknowledgements. This research was funded in part by NICHD R01 HD084214 (FTC) and the Ben \& Catherine Ivy Foundation (FTC). We are also thankful to GE Healthcare, PMOD Technologies LLC, BIK Therapeutics Inc. and the Stanford Cyclotron and Radiochemistry Facility for their kind assistance.

Ethics approval and consent to participate. The study protocol is part of a larger study NIH funded research study and approved by the Stanford University Institutional Review Board (IRB) and is compliant with federal, state, and local regulation on medical research (clinical trial registration no. NCT04308954). Exclusionary criteria included any contraindication for MRI scanning procedures (metal in body, braces, claustrophobia, etc.). Eligibility was assessed with a detailed intake questionnaire. The estimated completion time for this interview is approximately 30 minutes. Participants were screened for substance abuse, psychiatric conditions (bipolar disorder or schizophrenia), traumatic brain injury and BMI less than 18.5 for eligibility. All participants signed an assent form after reviewing with the study coordinator. In addition, participant's caregivers completed a study consent form. Both consent and assent forms were also approved by the Stanford University IRB.

\section{References}

Avants, B. B., Tustison, N., \& Song, G. (2009). Advanced Normalization Tools: The Insight Journal, V1.0.

Backes, M., Genç, B., Schreck, J., Doerfler, W., Lehmkuhl, G., \& von Gontard, A. (2000). Cognitive and behavioral profile of fragile X boys: Correlations to molecular data. American Journal of Medical Genetics, 95(2), 150-156. 
Bailey, D. B., Hatton, D. D., Skinner, M., \& Mesibov, G. (2001). Autistic Behavior, FMR1 Protein, and Developmental Trajectories in Young Males with Fragile X Syndrome. Journal of Autism and Developmental Disorders, 31(2), 165-174. https://doi.org/10.1023/A:1010747131386

Berry-Kravis, E., Knox, A., \& Hervey, C. (2011). Targeted treatments for fragile X syndrome. Journal of Neurodevelopmental Disorders, 3(3), 193. https://doi.org/10.1007/s11689-011-9074-7

Berry-Kravis, E., \& Potanos, K. (2004). Psychopharmacology in fragile X syndrome-Present and future. Mental Retardation and Developmental Disabilities Research Reviews, 10(1), 42-48. https://doi.org/10.1002/mrdd.20007

Brašić, J. R., Nandi, A., Russell, D. S., Jennings, D., Barret, O., Mathur, A., Slifer, K., Sedlak, T., Martin, S. D., Brinson, Z., Vyas, P., Seibyl, J. P., Berry-Kravis, E. M., Wong, D. F., \& Budimirovic, D. B. (2020). Reduced Expression of Cerebral Metabotropic Glutamate Receptor Subtype 5 in Men with Fragile X Syndrome. Brain Sciences, 10(12), 899. https://doi.org/10.3390/brainsci10120899

Gu, M., Hurd, R., Noeske, R., Baltusis, L., Hancock, R., Sacchet, M. D., Gotlib, I. H., Chin, F. T., \& Spielman, D. M. (2018). GABA editing with macromolecule suppression using an improved MEGA-SPECIAL sequence. Magnetic Resonance in Medicine, 79(1), 41-47. https://doi.org/10.1002/mrm.26691

Gousias, I. S., Rueckert, D., Heckemann, R. A., Dyet, L. E., Boardman, J. P., Edwards, A. D., \& Hammers, A. (2008). Automatic segmentation of brain MRIs of 2-year-olds into 83 regions of interest. Neurolmage, 40(2), 672-684. doi:10.1016/j.neuroimage.2007.11.034

Hammers, A., Allom, R., Koepp, M. J., Free, S. L., Myers, R., Lemieux, L., Mitchell, T. N., Brooks, D. J., \& Duncan, J. S. (2003). Three-dimensional maximum probability atlas of the human brain, with particular reference to the temporal lobe. Human Brain Mapping, 19(4), 224-247.

https://doi.org/10.1002/hbm.10123

Hunter, J., Rivero-Arias, O., Angelov, A., Kim, E., Fotheringham, I., \& Leal, J. (2014). Epidemiology of fragile $X$ syndrome: A systematic review and meta-analysis. American Journal of Medical Genetics Part A, 164(7), 1648-1658. https://doi.org/10.1002/ajmg.a.36511

Ichise, M., \& Ballinger, J. R. (1996). Editorial: SPECT imaging of dopamine receptors. J Nucl Med, 37, 1591-1595.

Laxman, D. J., Greenberg, J. S., DaWalt, L. S., Hong, J., Aman, M. G., \& Mailick, M. (2018). Medication use by adolescents and adults with fragile X syndrome. Journal of Intellectual Disability Research, 62(2), 94105. https://doi.org/10.1111/jir.12433

Palner, M., Beinat, C., Banister, S., Zanderigo, F., Park, J. H., Shen, B., Hjoernevik, T., Jung, J. H., Lee, B. C., Kim, S. E., Fung, L., \& Chin, F. T. (2016). Effects of common anesthetic agents on [18F]flumazenil binding to the GABAA receptor. EJNMMI Research, 6(1), 80. https://doi.org/10.1186/s13550-016-0235-2 
Smith, S. M., Jenkinson, M., Johansen-Berg, H., Rueckert, D., Nichols, T. E., Mackay, C. E., . .

Behrens, T. E. (2006). Tract-based spatial statistics: Voxelwise analysis of multi-subject diffusion data. Neurolmage, 31(4), 1487-1505. doi:10.1016/j.neuroimage.2006.02.024

Smith, S. M., Jenkinson, M., Woolrich, M. W., Beckmann, C. F., Behrens, T. E., Johansen-Berg, H., . . Matthews, P. M. (2004). Advances in functional and structural MR image analysis and implementation as FSL. Neurolmage, 23. doi:10.1016/j.neuroimage.2004.07.051

Tassone, F., long, K. P., Tong, T.-H., Lo, J., Gane, L. W., Berry-Kravis, E., Nguyen, D., Mu, L. Y., Laffin, J., Bailey, D. B., \& Hagerman, R. J. (2012). FMR1 CGG allele size and prevalence ascertained through newborn screening in the United States. Genome Medicine, 4(12), 100. https://doi.org/10.1186/gm401

The National Institue of Mental Health. (2020, August 17). Fragile X syndrome. Genetics Home Reference. https://ghr.nlm.nih.gov/condition/fragile-x-syndrome

Thurman, A. J., McDuffie, A., Kover, S. T., Hagerman, R. J., \& Abbeduto, L. (2015). Autism Symptomatology in Boys with Fragile X Syndrome: A Cross Sectional Developmental Trajectories Comparison with Nonsyndromic Autism Spectrum Disorder. Journal of Autism and Developmental Disorders, 45(9), 28162832. https://doi.org/10.1007/s10803-015-2443-4

Verkerk, A. J. M. H., Pieretti, M., Sutcliffe, J. S., Fu, Y.-H., Kuhl, D. P. A., Pizzuti, A., Reiner, O., Richards, S., Victoria, M. F., Zhang, F., Eussen, B. E., van Ommen, G.-J. B., Blonden, L. A. J., Riggins, G. J., Chastain, J. L., Kunst, C. B., Galjaard, H., Thomas Caskey, C., Nelson, D. L., ... Warren, S. T. (1991). Identification of a gene (FMR-1) containing a CGG repeat coincident with a breakpoint cluster region exhibiting length variation in fragile X syndrome. Cell, 65(5), 905-914. https://doi.org/10.1016/0092-8674(91)90397-H

\section{Tables}


Table 1

Study tasks and activities

\begin{tabular}{|ll|}
\hline Type of Activity & Activity \\
\hline Screening & Telephone Intake \\
\hline Consent & Electronic Consent \\
\hline Screening & Baseline Assessment \\
\hline MRI Preparation & MRI Games \\
\hline Consent & Full Study Consent/assent \\
\hline Cognitive & Stanford-Binet \\
\hline MRI Preparation & Practice MRI Session \\
\hline Behavioral & ADOS-2 \\
\hline Imaging & PET-MRI Scan Preparation \\
\hline Imaging & PET-MRI Scan \\
\hline Behavioral & VABS-II \\
\hline
\end{tabular}


Table 2

Non-displaceable binding potentials (BPND) of [18F]flumazenil, fractional anisotropy (FA), and mean diffusivity (MD) for selected regions of interest. FA values are $\mathrm{mm}^{2} / \mathrm{s}$. MD values are $\mathrm{mm}^{2} / \mathrm{s} \times 10^{-4}$.

\begin{tabular}{|c|c|c|c|c|}
\hline Region of interest & Hemisphere & $\mathrm{BP}_{\mathrm{ND}}$ & FA & MD \\
\hline Middle frontal gyrus & Left & 4.73 & $0.24 \pm 0.19$ & $11.47 \pm 6.38$ \\
\hline Middle frontal gyrus & Right & 4.85 & $0.24 \pm 0.18$ & $10.98 \pm 6.13$ \\
\hline Precentral gyrus & Left & 3.69 & $0.26 \pm 0.19$ & $10.19 \pm 5.42$ \\
\hline Precentral gyrus & Right & 3.66 & $0.26 \pm 0.18$ & $10.57 \pm 5.78$ \\
\hline Straight gyrus & Left & 5.03 & $0.19 \pm 0.11$ & $9.35 \pm 3.20$ \\
\hline Straight gyrus & Right & 4.93 & $0.20 \pm 0.14$ & $10.05 \pm 4.28$ \\
\hline Anterior orbital gyrus & Left & 4.74 & $0.29 \pm 0.22$ & $8.82 \pm 3.81$ \\
\hline Anterior orbital gyrus & Right & 4.69 & $0.27 \pm 0.20$ & $9.06 \pm 3.21$ \\
\hline Inferior frontal gyrus & Left & 4.71 & $0.23 \pm 0.16$ & $9.72 \pm 3.90$ \\
\hline Inferior frontal gyrus & Right & 4.86 & $0.21 \pm 0.16$ & $10.86 \pm 5.76$ \\
\hline Superior frontal gyrus & Left & 4.47 & $0.21 \pm 0.17$ & $11.78 \pm 6.56$ \\
\hline Superior frontal gyrus & Right & 4.32 & $0.22 \pm 0.17$ & $11.73 \pm 6.47$ \\
\hline Medial orbital gyrus & Left & 4.77 & $0.22 \pm 0.17$ & $9.44 \pm 3.07$ \\
\hline Medial orbital gyrus & Right & 4.65 & $0.24 \pm 0.18$ & $9.11 \pm 3.19$ \\
\hline Lateral orbital gyrus & Left & 4.69 & $0.23 \pm 0.21$ & $11.43 \pm 6.52$ \\
\hline Lateral orbital gyrus & Right & 4.62 & $0.24 \pm 0.20$ & $10.03 \pm 4.57$ \\
\hline Posterior orbital gyrus & Left & 4.44 & $0.25 \pm 0.17$ & $9.90 \pm 4.67$ \\
\hline Posterior orbital gyrus & Right & 4.34 & $0.27 \pm 0.17$ & $9.27 \pm 3.69$ \\
\hline Subgenual & Left & 4.26 & $0.34 \pm 0.24$ & $8.90 \pm 2.76$ \\
\hline Subgenual & Right & 3.60 & $0.34 \pm 0.22$ & $9.38 \pm 3.25$ \\
\hline Subcallosal area & Left & 4.11 & $0.20 \pm 0.09$ & $9.66 \pm 2.42$ \\
\hline Subcallosal area & Right & 3.95 & $0.20 \pm 0.11$ & $9.86 \pm 2.18$ \\
\hline Pre-subgenual & Left & 5.67 & $0.14 \pm 0.10$ & $9.78 \pm 3.69$ \\
\hline Pre-subgenual & Right & 4.48 & $0.17 \pm 0.10$ & $9.49 \pm 2.95$ \\
\hline Hippocampus & Right & 3.75 & $0.18 \pm 0.12$ & $10.39 \pm 3.76$ \\
\hline
\end{tabular}




\begin{tabular}{|c|c|c|c|c|}
\hline Region of interest & Hemisphere & $\mathrm{BP}_{\mathrm{ND}}$ & FA & MD \\
\hline Hippocampus & Left & 3.54 & $0.20 \pm 0.13$ & $10.19 \pm 3.57$ \\
\hline Amygdala & Right & 3.35 & $0.18 \pm 0.08$ & $9.53 \pm 4.32$ \\
\hline Amygdala & Left & 3.41 & $0.18 \pm 0.08$ & $10.79 \pm 6.24$ \\
\hline Anterior medial TL & Right & 3.74 & $0.21 \pm 0.15$ & $10.58 \pm 4.97$ \\
\hline Anterior medial TL & Left & 3.64 & $0.22 \pm 0.16$ & $11.66 \pm 6.74$ \\
\hline Anterior inferior lateral TL & Right & 4.47 & $0.18 \pm 0.11$ & $10.43 \pm 4.07$ \\
\hline Anterior inferior lateral TL & Left & 4.19 & $0.20 \pm 0.15$ & $10.23 \pm 4.44$ \\
\hline Parahippocampal gyrus & Right & 3.64 & $0.21 \pm 0.13$ & $10.00 \pm 4.85$ \\
\hline Parahippocampal gyrus & Left & 3.67 & $0.22 \pm 0.13$ & $9.90 \pm 4.77$ \\
\hline Superior temporal posterior gyrus & Right & 4.49 & $0.23 \pm 0.17$ & $10.11 \pm 4.66$ \\
\hline Superior temporal posterior gyrus & Left & 4.69 & $0.23 \pm 0.18$ & $10.08 \pm 4.62$ \\
\hline Middle \& inferior temporal gyrus & Right & 4.24 & $0.26 \pm 0.17$ & $8.57 \pm 2.50$ \\
\hline Middle \& inferior temporal gyrus & Left & 4.22 & $0.25 \pm 0.17$ & $8.31 \pm 1.90$ \\
\hline Fusiform gyrus & Right & 4.17 & $0.23 \pm 0.14$ & $8.95 \pm 3.00$ \\
\hline Fusiform gyrus & Left & 4.42 & $0.23 \pm 0.14$ & $8.73 \pm 3.50$ \\
\hline Posterior TL & Left & 4.32 & $0.27 \pm 0.19$ & $9.08 \pm 3.82$ \\
\hline Posterior TL & Rsight & 4.38 & $0.27 \pm 0.20$ & $8.95 \pm 3.87$ \\
\hline Superior temporal anterior gyrus & Left & 4.39 & $0.20 \pm 0.16$ & $12.51 \pm 7.04$ \\
\hline Superior temporal anterior gyrus & Right & 4.54 & $0.18 \pm 0.12$ & $10.96 \pm 5.74$ \\
\hline Postcentral gyrus & Left & 4.04 & $0.25 \pm 0.19$ & $10.18 \pm 4.93$ \\
\hline Postcentral gyrus & Right & 3.95 & $0.26 \pm 0.20$ & $10.96 \pm 6.28$ \\
\hline Superior parietal gyrus & Left & 4.20 & $0.24 \pm 0.19$ & $10.62 \pm 5.15$ \\
\hline Superior parietal gyrus & Right & 3.95 & $0.24 \pm 0.19$ & $10.73 \pm 5.70$ \\
\hline Inferiolateral remainder of parietal lobe & Left & 4.21 & $0.24 \pm 0.19$ & $10.11 \pm 5.27$ \\
\hline Inferiolateral remainder of parietal lobe & Right & 4.20 & $0.22 \pm 0.18$ & $11.21 \pm 6.06$ \\
\hline Lateral reminder of occiptal lobe & Left & 3.95 & $0.25 \pm 0.18$ & $8.84 \pm 3.14$ \\
\hline Lateral reminder of occiptal lobe & Right & 3.87 & $0.25 \pm 0.18$ & $8.72 \pm 3.21$ \\
\hline Lingular gyrus & Left & 4.87 & $0.22 \pm 0.16$ & $8.61 \pm 3.44$ \\
\hline
\end{tabular}




\begin{tabular}{|c|c|c|c|c|}
\hline Region of interest & Hemisphere & $\mathrm{BP}_{\mathrm{ND}}$ & FA & MD \\
\hline Lingular gyrus & Right & 4.77 & $0.22 \pm 0.17$ & $8.52 \pm 3.38$ \\
\hline Cuneus & Left & 4.69 & $0.18 \pm 0.15$ & $9.82 \pm 4.49$ \\
\hline Cuneus & Right & 4.85 & $0.18 \pm 0.14$ & $9.49 \pm 4.21$ \\
\hline Caudate nucleus & Left & 1.29 & $0.18 \pm 0.10$ & $9.87 \pm 5.25$ \\
\hline Caudate nucleus & Right & 1.43 & $0.18 \pm 0.09$ & $9.40 \pm 4.46$ \\
\hline Nucleus accumbens & Left & 3.75 & $0.16 \pm 0.06$ & $7.85 \pm 1.00$ \\
\hline Nucleus accumbens & Right & 4.02 & $0.16 \pm 0.06$ & $8.20 \pm 1.27$ \\
\hline Putamen & Left & 1.90 & $0.23 \pm 0.15$ & $7.57 \pm 0.69$ \\
\hline Putamen & Right & 2.02 & $0.23 \pm 0.13$ & $7.44 \pm 0.73$ \\
\hline Thalamus & Left & 1.99 & $0.32 \pm 0.11$ & $9.27 \pm 4.93$ \\
\hline Thalamus & Right & 1.97 & $0.31 \pm 0.12$ & $9.24 \pm 5.36$ \\
\hline Pallidum & Left & 1.86 & $0.35 \pm 0.16$ & $8.38 \pm 1.41$ \\
\hline Pallidum & Right & 1.89 & $0.35 \pm 0.16$ & $8.29 \pm 1.38$ \\
\hline Corpus callosum & & 2.10 & $0.57 \pm 0.28$ & $9.49 \pm 4.31$ \\
\hline Substantia nigra & Left & 0.49 & $0.42 \pm 0.19$ & $11.64 \pm 7.70$ \\
\hline Substantia nigra & Right & 0.38 & $0.47 \pm 0.20$ & $12.29 \pm 7.92$ \\
\hline Insula & Left & 3.94 & $0.32 \pm 0.20$ & $8.89 \pm 3.20$ \\
\hline Insula & Right & 4.14 & $0.31 \pm 0.20$ & $8.81 \pm 2.82$ \\
\hline Anterior cingulate gyrus & Left & 4.75 & $0.13 \pm 0.09$ & $11.30 \pm 4.91$ \\
\hline Anterior cingulate gyrus & Right & 4.35 & $0.16 \pm 0.12$ & $10.42 \pm 4.35$ \\
\hline Posterior cingulate gyrus & Left & 4.84 & $0.18 \pm 0.16$ & $10.57 \pm 4.83$ \\
\hline Posterior cingulate gyrus & Right & 4.58 & $0.20 \pm 0.16$ & $9.90 \pm 4.23$ \\
\hline Cerebellum & Left & 2.46 & $0.26 \pm 0.15$ & $9.20 \pm 5.73$ \\
\hline Cerebellum & Right & 2.49 & $0.27 \pm 0.15$ & $8.60 \pm 5.61$ \\
\hline Brainstem & & 0.71 & $0.40 \pm 0.21$ & $12.30 \pm 10.02$ \\
\hline FrontalHorn & Right & 0.59 & $0.30 \pm 0.24$ & $23.97 \pm 10.69$ \\
\hline FrontalHorn & Left & 0.55 & $0.36 \pm 0.27$ & $21.54 \pm 10.82$ \\
\hline TemporaHorn & Right & 2.00 & $0.35 \pm 0.17$ & $11.41 \pm 4.46$ \\
\hline
\end{tabular}




\begin{tabular}{|lllll|}
\hline Region of interest & Hemisphere & BP $_{\text {ND }}$ & FA & MD \\
\hline TemporaHorn & Left & 1.63 & $0.38 \pm 0.18$ & $9.28 \pm 3.53$ \\
\hline Third ventricle & & 1.26 & $0.15 \pm 0.06$ & $23.12 \pm 8.05$ \\
\hline White matter & & 1.54 & $0.39 \pm 0.18$ & $8.04 \pm 3.06$ \\
\hline
\end{tabular}

\section{Figures}

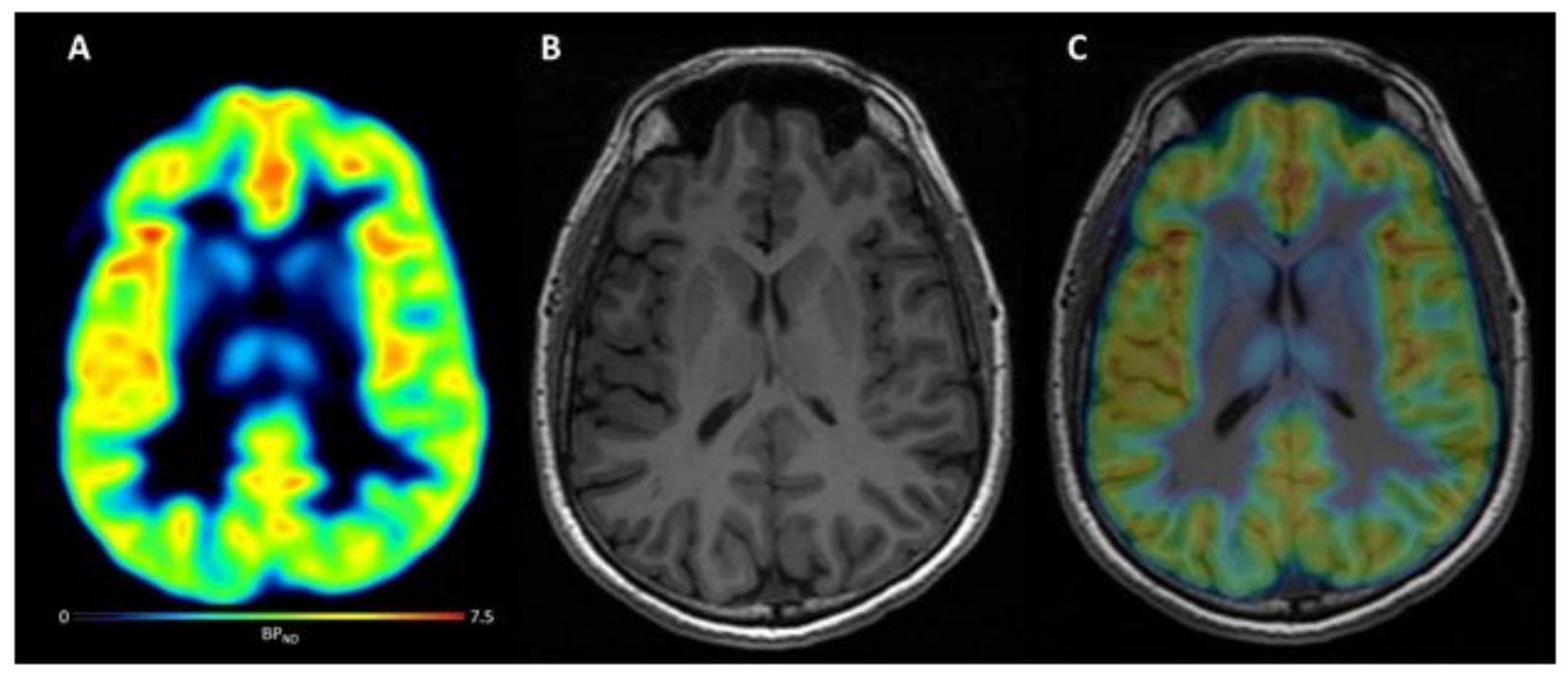

\section{Figure 1}

Parametric PET image and structural MRI. Parametric image of non-displaceable binding potential (BPND) of [18F]flumazenil (A), structural MRI (B) and fused PET-MRI (C). The color bar for the PET image reflects the minimum and maximum BPND values. 


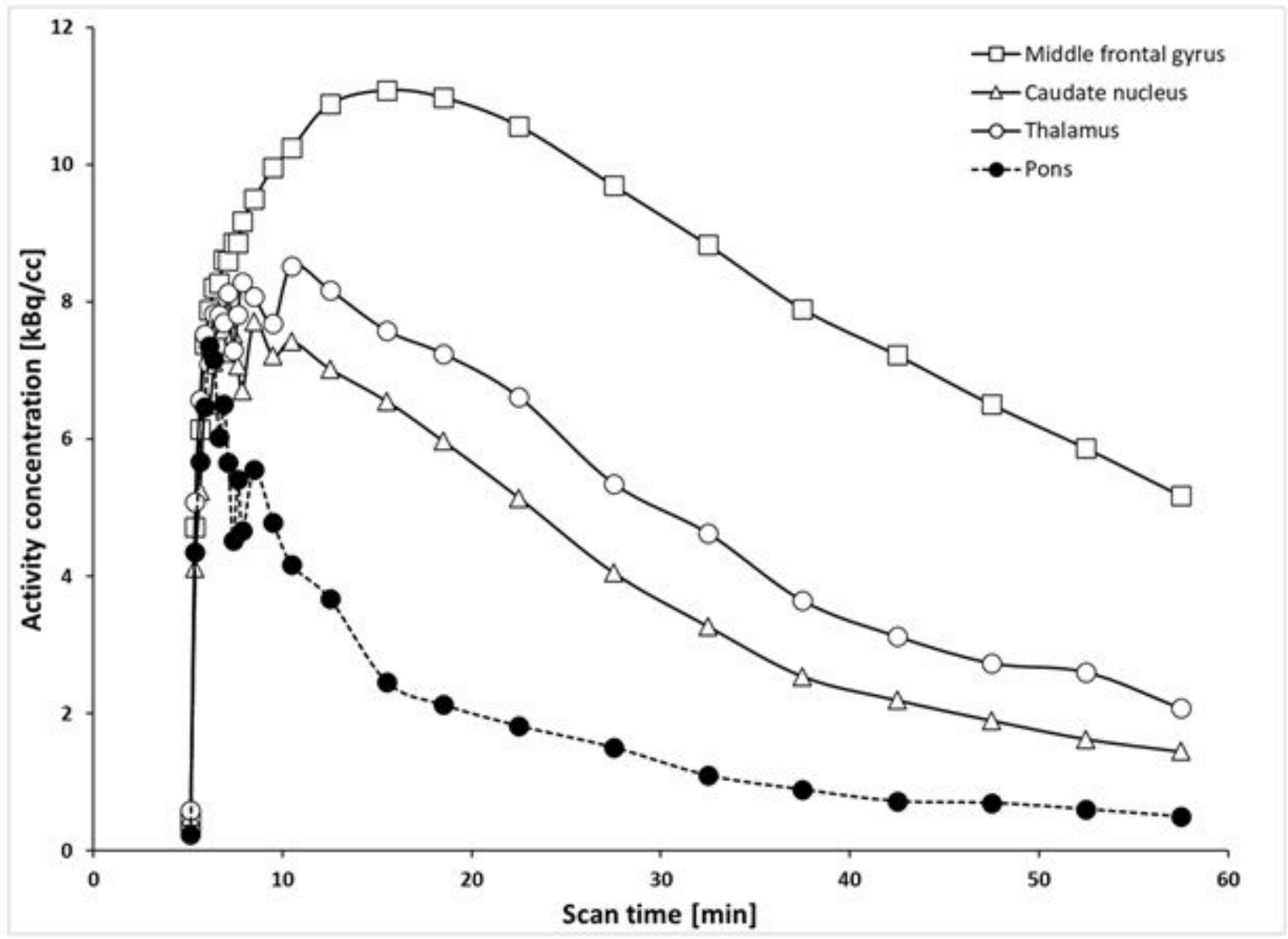

Figure 2

Tissue time-activity curves. Tissue time-activity curves (TACs) for four selected regions of interest: middle frontal gyrus, caudate nucleus, thalamus and pons. TACs shown are corrected for radioactive decay. 

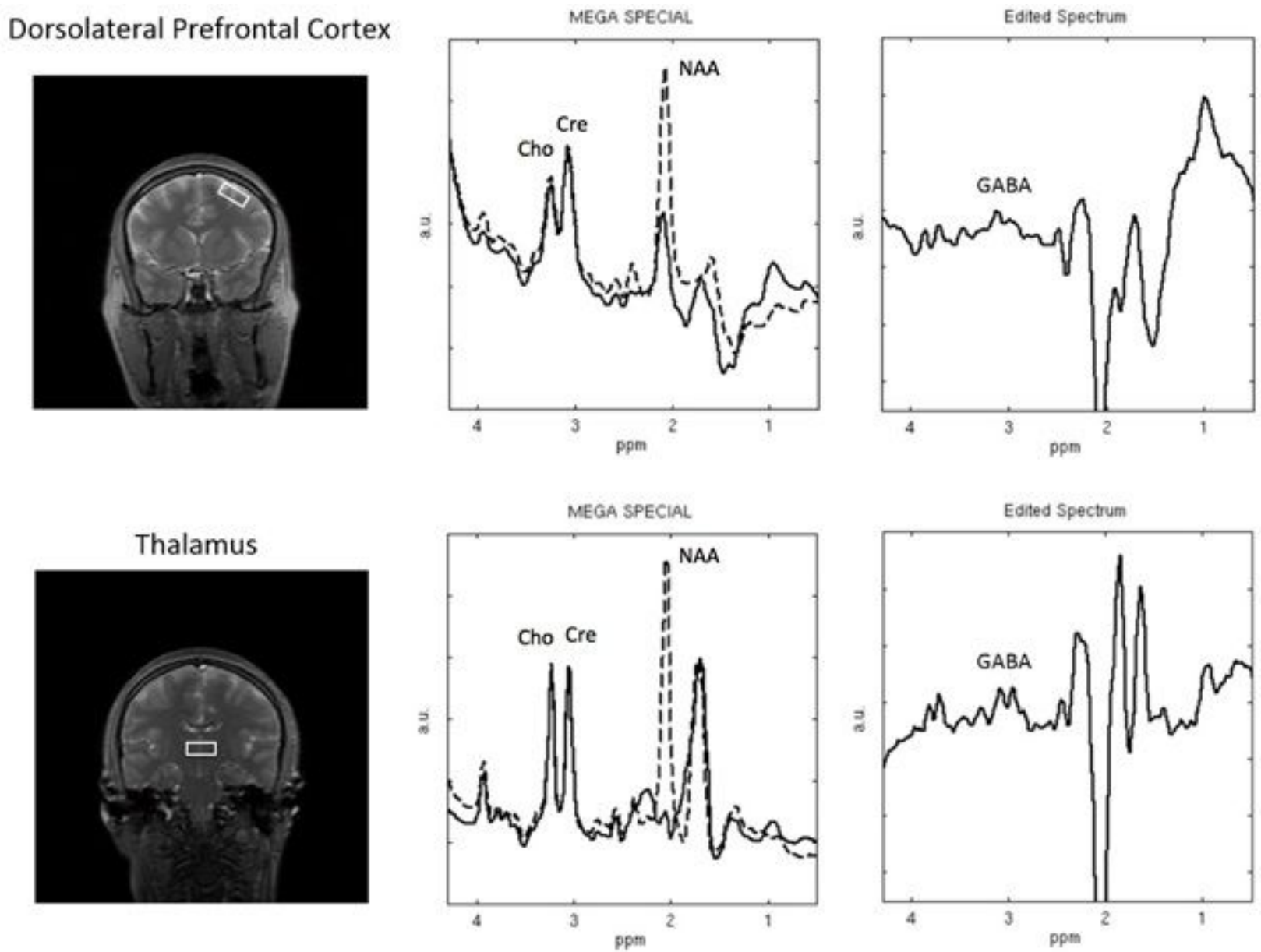

Figure 3

MR spectra with GABA editing ON and OFF and the edited spectra from voxels in the thalamus and DLPFC of a patient with Fragile-X. MRS data were acquired on a GE PET-MR 3T scanner simultaneously with the PET data acquisition using an improved MEGA-SPECAL sequence with TE/TR=80/2000ms, 15 min acquisition/voxel 


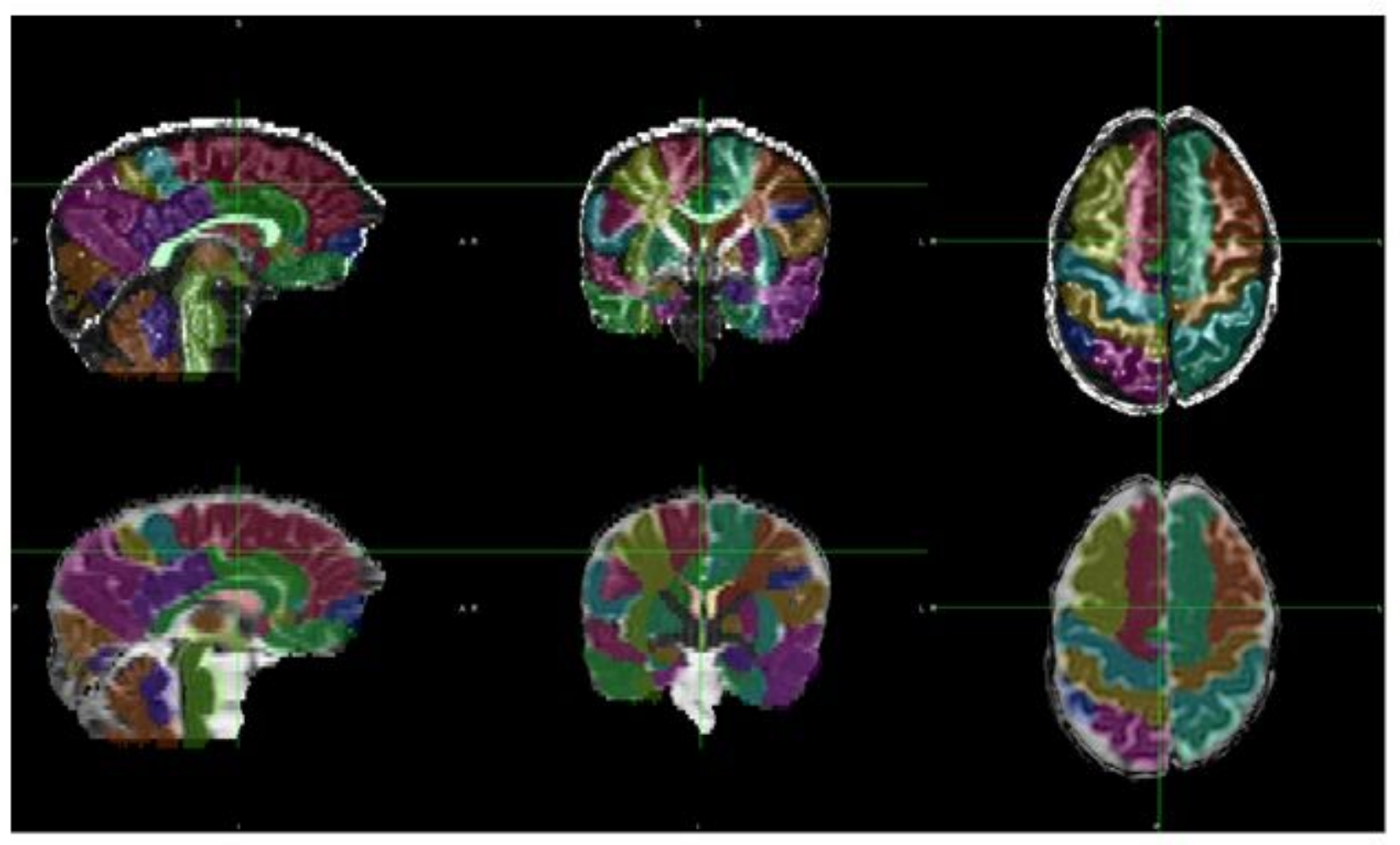

\section{Figure 4}

Regions of interest (ROI) overlaid on maps of fractional anisotropy (FA) (top row) and mean diffusivity (MD) (bottom row). Color-coded ROls were defined based on the Hammersmith atlas. 